\title{
Mesenchymal Stem Cells Derived from Wharton's Jelly and their Potential for Cardio-Vascular Tissue Engineering
}

\author{
O.V. Semenov ${ }^{1}$ and C. Breymann ${ }^{*}, 2$ \\ ${ }^{1}$ Swiss Transfusion SRC, Laupenstrasse 37, CH-3001 Bern, Switzerland \\ ${ }^{2}$ Department of Obstetrics, Feto Maternal Haematology Research Unit, University Hospital Zurich/Switzerland
}

\begin{abstract}
Experimental results accumulated during last decade suggest that human perinatal tissues such as placenta, fetal membranes, and umbilical cord, as well as perinatal fluids such as, amniotic fluid and umbilical cord blood, harbour different amounts of multipotent precursor cells, called extra-embryonic mesenchymal stem cells (EE-MSCs). Perinatal EE-MSCs represent an intermediate cell type between pluripotent embryonic stem cells (ESCs) and multipotent MSCs derived from variety of postnatal human tissues, such as bone marrow, fat, dental pulp, etc. Multipotent mesenchymal cells obtained from connective Wharton's Jelly tissue of umbilical cord (WJ-MSCs) currently emerged as particularly interesting type of perinatal EE-MSCs, related for therapeutical applications and cryobanking. These cells are easily assessable for isolation, possess fetal karyotype, and hold very active growth potential. Due to their unique developmental position WJ-MSCs exhibit specific phenotype which combines some markers expressed by postnatal bone marrowderived MSCs, such as CD73, CD90, and CD105 with some markers typically expressed by ESCs, such as Oct-3/4, Sox2, and Nanog. In terms of plasticity WJ-MSCs demonstrate the potential for differentiation towards mesodermal and ectodermal lineages in vitro and in vivo. Additionally, extraembryonic tissues are normally discarded after birth and the isolation of WJ-MSCs is free of ethical concerns. In this work we aim to review previously obtained experimental results, discuss different aspects concerning plasticity and immunomodulatory characteristics of WJ-MSCs, and evaluate the potential of these cells for biomedical and clinical applications.
\end{abstract}

Keywords: Mesenchymal stem cells, wharton's jelly, tissue engineering.

\section{EXTRAEMBRYONIC PERINATAL TISSUES - NEW PUTATIVE SOURCES OF STEM CELLS}

Emerging evidence indicates that different postnatal human tissues harbour small amounts of mesenchymal stem cells (MSCs), which have the potential to give rise to all cell types of the source tissue [1,2]. Previously isolated from human bone marrow, currently MSC harvest sites include the muscles, fat, brain, cartilage, and dental pulp [3-5].

Clinical expectations associated with MSCs are derived from three functional characteristics of these cells: the ability (I) for tissue reparation through direct or paracrine effects, (II) for immunomodulation, and (III) to support cell engraftment. Different research efforts have demonstrated that adult MSCs have a broad therapeutic potential primarily due to their ability to regenerate tissue by differentiating towards multiple adult cell types under appropriate in vivo and in vitro conditions $[6,7]$. Moreover, a growing body of data demonstrates the potential use of ex vivo-expanded adult MSCs in modulating immunologic response through interactions with immune cells such as T- and B-lymphocytes, natural killer cells, and dendritic cells [8-11]. These immunosuppressive properties of MSCs characterize them as promising candidates for treating immunologic disorders like Crohn's disease. Additionally, there is increased potential for

*Address correspondence to this author at the University Hospital Zurich, Frauenklinikstr. 10, CH-8091 Zurich; Tel: +41 44255 5148;

E-mail: christian.breymann@usz.ch
MSC use in decreasing occurrences of Graft-versus-Host disease after allogenic haematopoietic stem cell (HSC) and solid organ-transplantation [12, 13].

Earlier source tissues of stem cells were classified into two general categories dependent on the timepoint of ontogenesis: embryonic and postnatal also called adult tissues. In the last decade, the list of putative human stem cell sources was amended to include human perinatal extra-embryonic tissues [14, 15]. Generally, human extra-embryonic tissues are represented by different parts of the placenta, fetal membranes (amnion and chorion), and umbilical cord [1618]. Furthermore, it has been shown that extra-embryonic MSCs can be isolated from umbilical cord blood (UCB) and amniotic fluid (AF) [19-21].

Extra-embryonic perinatal MSCs represent an intermediate stem cell type that partially combines some pluripotent properties of embryonic stem cells (ESCs) with some multipotent properties of adult postnatal mesenchymal stem cells (MSCs) [15]. Due to its close ontogenetic relationship with embryonic stem cells, extra-embryonic tissue-derived MSCs have immunoprivileged characteristics, possess a broader multipotent plasticity, and proliferate faster than adult postnatal MSCs [14, 15]. Moreover, because extraembryonic tissues are normally discarded after birth, these cells could be isolated while effectively avoiding ethical concerns [14].

Previously, cells obtained from cord blood, amniotic fluid, and fetal membranes were used in perinatal medicine for invasive diagnostic purposes, such as sex determination, 
or for the detection of fetal infections, rare metabolic disorders, and genetic diseases such as chorioamnionitis, osteogenesis imperfecta, and trisomy-21. The use of peri-natal stem cells in treating haematological and metabolic diseases dates back to the 1980s, when umbilical cord blood was successfully conducted to treat a patient with Fanconi anemia using UCB from human leukocyte antigen (HLA) matched sibling donor [22]. Since then, there have been over 10.000 successful UCB transplants conducted worldwide [23]. The 1990s demonstrated the usability of perinatal extra-embryonic MSCs in tissue engineering applications, just after Langer's and Vacanti brothers' introduction of the concept of regenerative medicine [24, 25].

\section{HUMAN PLACENTAL ANATOMY AND FUNCTION}

Functionally, the human placenta can be seen as a foetomaternal organ that segregates maternal and foetal circulation while serving as a protective immunological barrier and site of oxygen, nutrient, and metabolite exchange [26-28]. It also has multiple endocrine functions and participates in the production of steroid and protein hormones, such as progesterone and estrogen as well as placental lactogen and chorionic gonadotropin $[27,28]$. Progesterone is responsible for the maintenance of endometrial lining during pregnancy and prevention of preterm labor by reducing of myometrial contractions [27, 28]. Estrogen is responsible for stimulation of uterine growth to accommodate growing fetus [27, 28]. Placental lactogen regulates maternal glucose, fat, and protein levels, so that it is always available for the growing fetus [27, 28]. Chorionic gonadotropin is reduces maternal immunologic response and protecting placenta against rejection $[27,28]$.

Anatomically, the human placenta at full term represents a circular discus-like organ, with an average diameter of about $22 \mathrm{~cm}$, thickness of $2.5 \mathrm{~cm}$, and weight of $470 \mathrm{~g}$ [2729]. Structurally, it can be divided into foetal and maternal parts, termed surfaces. These surfaces merge and form the smooth chorion as well as the foetal membranes. A chorionic plate with an inserted umbilical cord characterizes the foetal surface. This chorionic plate is covered by amnion, which is composed of a single epithelial layer and an avascular amniotic mesenchyme that is weakly attached to chorionic mesenchyme. The chorionic mesenchyme contains vessels that are linked with umbilical cord vessels on one side and villous tree vessels and veins on another side [27-30].

The maternal surface, termed the basal plate, is typified by a sizable extracellular matrix and fibrinoids. It consists of a mixture of fetal extra-villous trophoblastic cells with maternal cells of the uterine decidua, such as decidual stromal cells, natural killer cells, macrophages, and other immune cell types [30]. The basal plate of a full-term placenta is subdivided into 10 to 40 slightly elevated regions called maternal lobes. Maternal lobe location corresponds with the location of 60 to 70 foetal lobes [27-30].

The umbilical cord is an elastic cord connecting the foetus and placenta during pregnancy. Functionally, the cord protects enclosed vessels from compression, torsion, and bending while providing a bidirectional, foeto-maternal blood circulation (Fig. 1). A mature umbilical cord has a mean length of 50 to $70 \mathrm{~cm}$ (though the range spans 25 to
$100 \mathrm{~cm}$ ), in vivo diameter of $12 \mathrm{~mm}$, and weight of $100 \mathrm{~g}$ (ranging from 40 to $150 \mathrm{~g}$ ) [31, 32].

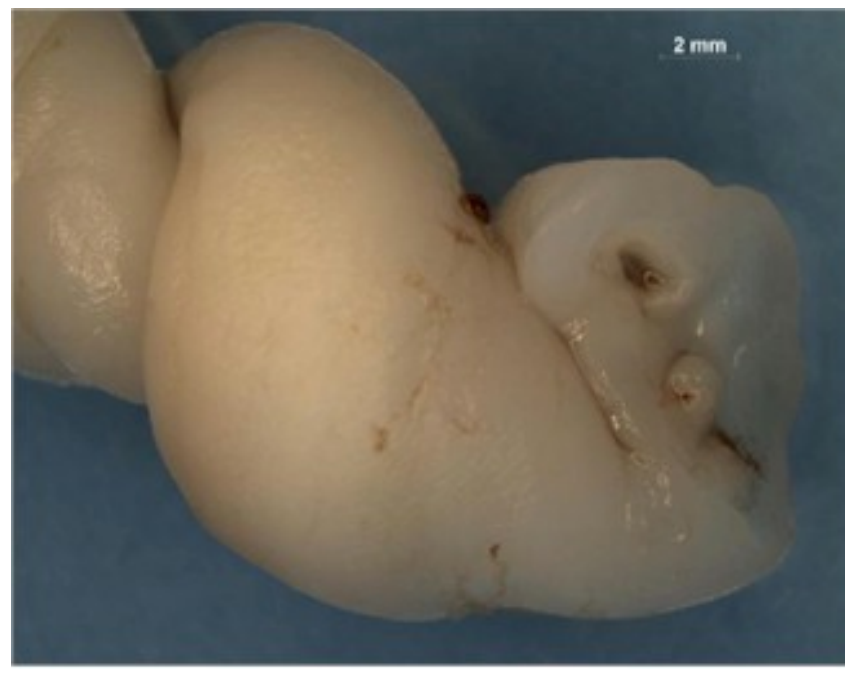

Fig. (1). Anatomy of the normal human umbilical cord (macroscopic image).

Anatomically, the umbilical cord consists of two umbilical arteries and one umbilical vein, both embedded within a specific mucous proteoglycan-rich matrix, known as Wharton's jelly, which is then covered by amniotic epithelium (Fig. 2). The jelly-like connective tissue is composed of collagen fibres of Type I and III and a glycosaminoglycan (GAG) - containing jelly-like intercellular substance. The collagen creates a three-dimensional network and is in loose contact with amorphic intercellular substance contains mucopolysaccharids, such as hyaluronic acid, and carbohydrates with glycosyl and mannosyl terminals and acid groups [33, 34].

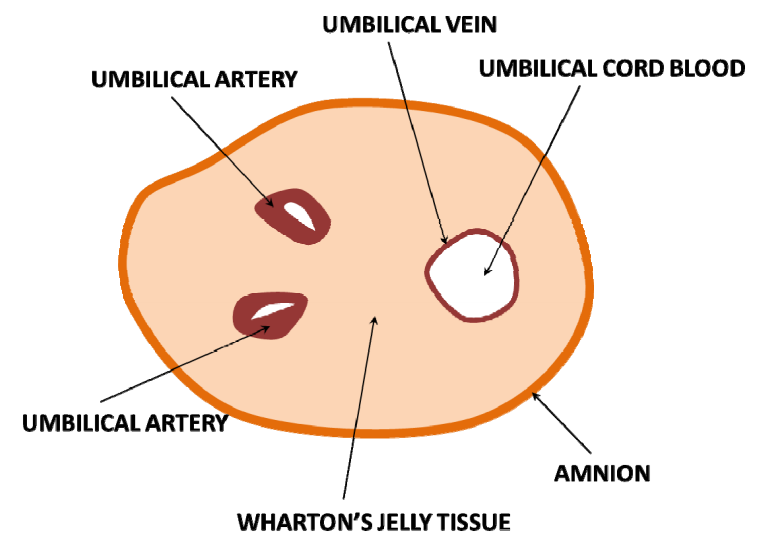

Fig. (2). Structure of umbilical cord blood (schematic image).

\section{THE UMBILICAL CORD IS A SOURCE OF MESEN- CHYMAL STEM CELLS}

Results showing that Wharton's jelly contains a multipotent fibroblast-like mesenchymal cell population were first obtained more than ten years ago [35]. Previously, these cells were termed as "umbilical cord matrix stem cells" to distinguish them from endothelial cells isolated from umbilical vein (HUVEC) as well as from late outgrowth 
endothelial cells (OECs) and mesenchymal cells (UCBMSCs) isolated from umbilical cord blood [19, 36, 37]. Though they have lately been termed Wharton's jellyderived mesenchymal stem cells (WJ-MSCs) [38-40].

Currently, WJ-MSCs are isolated from three regions: the perivascular zone, the inter-vascular zone, and the subamnion [41]. Fine structural, immuno-histochemical, and functional analysis performed in vitro show significant differences in the number and nature of cells among subamniotic, inter-vascular, and perivascular regions [42, 43]. These findings lead to hypothesis that these regions might be originating from different pre-existing formations [44]. At the same time it is still unknown whether fibroblast-like cells obtained from different compartments of umbilical cords represents different populations and possess different properties [42]. They express similar surface markers, suggesting that they are all of MSC origin [41]. However, it has been shown that the WJ-MSCs located close to amniotic surface retaining better ability to proliferate, whereas WJMSCs with broader differentiation potential were found in closer proximity to the umbilical vessels $[42,43]$.

The most common methods for WJ-MSC isolation are based on the use of explant-culture or enzymatic digestion techniques $[45,46]$. Normally freshly obliterated $15-20 \mathrm{~cm}$ umbilical cords will be immediately transported in the laboratory and the umbilical arteries and veins will be removed. After that Whartons's jelly tissue will be excised and minced using a scalpel or surgical scissors, after which the small pieces of tissue will be either directly plated on a tissue culture polystyrene petri dish as explants or additionally digested using proteolytic enzymes (such as collagenase and hyaluronidase). If a digestion step was performed, cell suspensions will be normally filtered through 70-100 um sieves, resuspended in culture medium, and plated out in culture flasks [41, 47]. The advantage of enzymatic digestion technique is related with the purity of obtained cell culture. Normally, if digestion step has been performed, the risk of WJ-MSC contamination with other cell types, such as blood cells or endothelial cells, as well as tissue debris is reduced [41]. Freshly isolated WJ-MSCs normally demonstrate fibroblast-like appearance during the first culture period (10-15 days) until first passage (Fig. 3). Population doubling time which is approximately 60-85 hours at early passages dramatically declines at late passages [42].

On one hand, WJ-MSCs meet the minimal criteria for defining adult MSCs - morphologically, they resemble plastic adherent adult postnatal MSCs, which are able to selfrenew, and can be expanded as an in vitro culture [48]. Phenotypically, WJ-MSCs express typical mesenchymal stem cell markers like CD10, CD13, CD29, CD44, CD73, CD90, and CD105. At the same time, they do not express markers of the hematopoietic lineage, such as CD34, and CD45 [49, 50]. In terms of plasticity, WJ-MSCs are multipotent and can be induced to form adipose tissue, bone, cartilage, skeletal muscle cells, cardiomyocyte-like cells, and neural cells $[41,44]$.

In another regard, WJ-MSCs possess the typical properties of extra-embryonic perinatal MSCs. Generally properties of WJ-MSCs are very similar with the properties of placenta-derived (PD-MSCs) and properties of cord blood-derived (UCB-MSCs) [16, 19]. First, similarly to PDMSCs and UCB-MSCs, WJ-MSCs are of mesenchymal origin and possess multipotent plasticity. They have a greater

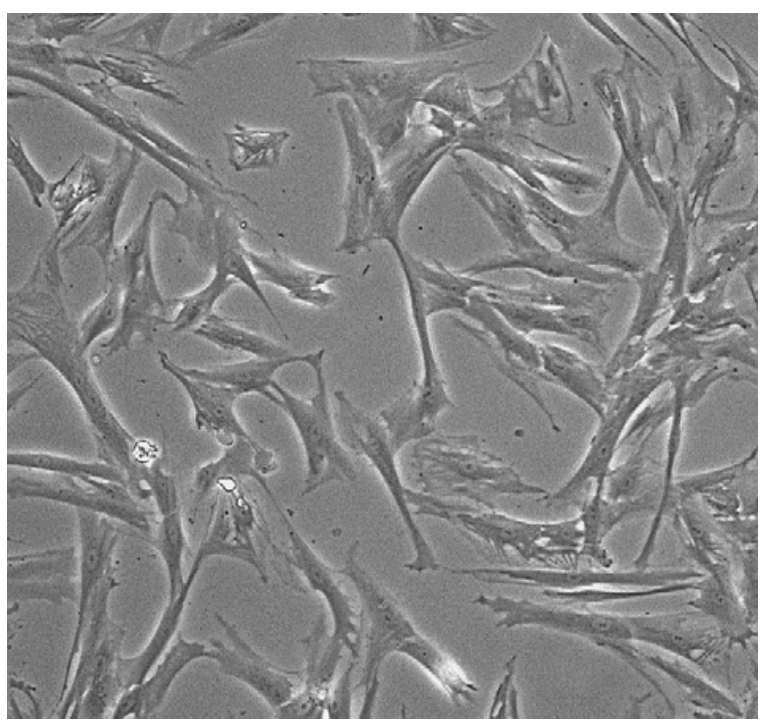

Fig. (3). Typical fibroblast-like morphology of WJ-MSCs expanded in vitro.

expansion potential in vitro $(80$ and more population doublings) than adult postnatal MSCs. Second, they are characterized by a much lower expression of HLA-class I, and lack of expression of HLA class-II, surface markers than adult postnatal MSCs, such as bone marrow-derived MSCs [41]. Third, similarly to PD-MSCs and UCB-MSCs, WJMSCs possess a broader differentiation potential as adult MSCs. Very recent data shows that WJ-MSCs can be differentiated towards endoderm-derived tissues, such as those of the pancreas and liver [51, 52]. Analogous to umbilical cord blood-derived MSCs, WJ-MSCs also improve properties of osteogenesis and neurogenesis in vitro [42]. Unlike postnatal MSCs and PD-MSCs, WJ-MSCs consistently express embryonic stem cell markers like Oct-4, Sox-2, and Nanog. They also express markers of pluripotency, such as SSeA-4 and Tra-1-60. Additionally, WJ-MSCs show much broader characteristics with respect to immunomodulation as postnatal MSCs. For example, it has been shown that WJMSCs inhibit T-cell proliferation during mixed lymphocyte assay and tolerated allogenic transplant [12]. Based on these unique properties Wharton's jelly MSCs can be characterized as an amenable, plentiful, and inexpensive source of multipotent MSCs with promising potential for use in regenerative medicine applications [48].

\section{APPLICATION OF WJ-MSCS IN CARDIOVASCU- LAR TISSUE ENGINEERING}

Due to the limited regenerative potential of human cardiovascular system, development of functional replacements that support the regeneration of damaged or diseased cardiovascular tissues, especially for newborn and pediatric patients, is critical. Surgical treatment is commonly based on non-autologous valves or conduits, which have distinct disadvantages including obstructive tissue ingrowths and calcification of the implant $[53,54]$. These limitations and the lack of growth typically necessitate re-operations of 
pediatric patients with cardiovascular defects, which are consequently associated with an increased risk of morbidity and mortality each time. Therefore, cardiovascular fetal tissue engineering focuses on the in vitro fabrication of autologous, living tissue with the potential for regeneration of heart muscle. This promising scientific field aims to address the currently unmet medical need of growing replacements, particularly those for congenital malformation repair [55].

Two adult cell types routinely used for such fabrication of cardiovascular tissues are (I) cells with the capacity to form an extracellular matrix, commonly myofibroblasts, and (II) endothelial cells with antithrombogenic characteristics. Seeding cells onto three-dimensional scaffolds is sequential - myofibroblasts are seeded first, followed by endothelial cells [56]. Due to their better self-renewal capacity, broader plasticity, and immunomodullatory properties comparable to adult fibroblasts, tissue-engineered constructs based on perinatal stem cells in general (and particularly WJ-MSCs) could be an attractive alternative to classical cardiovascular substitutes. WJ-MSCs would be ideal for tissue-engineered constructs as they are autologous, can be harvested through minimally invasive means, possess excellent growth capacities, and are able to form an optimal neo-matrix with excellent mechanical properties. Furthermore, endothelial cells, necessary for the generation of cardio-vascular substitutes, could be isolated either from umbilical cord vessels or umbilical cord blood $[57,58]$.

The feasibility of using WJ-MSCs as an alternative, autologous cell source for cardiovascular tissue engineering as well as their feasibility in developing pulmonary artery conduits was investigated starting from 2002 [59-61]. Scientists concluded that the in vitro fabrication of tissueengineered human pulmonary conduits was feasible utilizing human WJ-MSCs within a biomimetic culture environment. Interestingly, the morphologic and mechanical features closely approximated those of a native human pulmonary artery. Human WJ-MSCs demonstrated excellent growth properties representing a new, readily available cell source for tissue engineering without sacrificing intact vascular donor structures.

In 2004, researchers studied various umbilical tissues as potential sources for tissue engineering [62]. The study, performed on adult humans, evaluated cells isolated from the umbilical cord artery (UCA), umbilical cord vein (UCV), whole umbilical cord (UCC) and saphenous vein segments (VC) as alternative autologous cell sources for cardiovascular applications. Cells from the UCA, UCV and UCC demonstrated excellent cell growth properties comparable to VC. Following isolation, all three cell groups showed myofibroblast-like morphology and characteristics by staining positive for alpha-smooth muscle actin (ASMA) and vimentin. Histology and immunohistochemistry of seeded polymers showed good tissue and extracellular matrix formation containing collagen I, III, and elastin. Transmission electron microscopy showed viable myofibroblasts and the deposition of collagen fibrils and progressively-growing tissue formation, with a confluent surface, was observed via scanning electron microscopy. UCA, UCV, UCC and VC tissueengineered constructs did not differ in their mechanical properties. Cell growth, morphology, characteristics and tissue formation were comparable between $\mathrm{UCA}, \mathrm{UCV}$, $\mathrm{UCC}$ and $\mathrm{VC}$ leading to the conclusion that the tissue engineering of cardiovascular constructs by using cells from the UCA, UCV, and UCC is feasible in an in vitro environment.

In 2005, the first results related to fabrication of living patches engineered from WJ-MSCs and endothelial progenitor cells (EPCs) were published [63]. Scientists observed seeded patches representing layered, viable, tissue-like structures. The WJ-MSCs in the newly formed tissues expressed myofibroblast markers, such as desmin and ASMA. The EPCs derived neo-endothelia showed constant endothelial phenotypes (CD 31, vWF). Major constituents of ECM such as collagen and proteoglycans were biochemically detected and stress-strain properties of the patches showed features of native-analogous tissues.

One year later, in 2006, the preliminary results were reported for living autologous heart valves based on WJMSCs $[64,65]$. In this study, biologically-active heart valve leaflets were engineered using prenatally available human umbilical cord-derived progenitor cells as the only cell source. WJ-MSCs and umbilical cord blood-derived EPCs were subsequently seeded on biodegradable scaffolds and cultured in a biomimetic system under biochemical or mechanical stimulation or both. Depending on the stimulation, the leaflets showed mature, layered tissue formation with functional endothelia and extracellular matrix production comparable with that of native tissues. This demonstrates the feasibility of heart valve leaflet fabrication from prenatal umbilical cord-derived progenitor cells as a further step in overcoming the lack of living autologous replacements with growth and regeneration potential for the repair of congenital malformation [55].

The general concept of WJ-MSC-based cardiovascular tissue engineering has also been validated in large animal studies [66]. Precisely, completely autologous, living trileaflet heart valves generated using human WJ-MSCs seeded on biodegradable matrices have been successfully implanted in growing sheep models for up to 20 weeks. These valves showed good functional performance as well as structural and biomechanical characteristics strongly resembling those of native semilunar heart valves.

In 2010, the procedure for microencapsulating WJ-MSCs has been reported [67]. This study describes the functional properties in terms of secretive profiles of both free and encapsulated WJ-MSCs. Interestingly, microencapsulation did not alter the morphology and viability of the WJ-MSCs and the encapsulation procedure represents a promising strategy for in vivo utilization of WJ-MSCs for possible applications in cardio-vascular tissue engineering and biomedicine. Immuno-protective capsules or devices will be used in regenerative medicine as vehicles for the delivery of different therapeutic agents as well as cells to the injury cite. Because of their specific structural characteristics, such as spherical configuration and small size, microcapsules have much better surface-to-volume ratios than macro-vehicles. Second, microcapsules allow precise tailoring of permeability to allow diffusion of anabolic compounds (oxygen, glucose, etc.) and of cell-derived products (carbon dioxide, lactate, hormones, etc.) while, simultaneously excluding immuno-globulins. Third, microcapsules minimize the overall risk of immuno- 
protection failure by using thousands of them instead of a single large macro-capsule. Fourth, they can be injected directly or transplanted with minimal-invasive surgery into the different tissue and organs [68].

Very recently, in 2011 development of myocardial patches based on WJ-MSCs incorporated in 3D aligned microfibers desired for potential treatment of myocardial infarctions and improvement of long term cardiac tissue functions has been described [69]. The experimental 3D construct design is based on two biodegradable macroporous tubes, which allow transport of growth media to the cells within the construct itself, and cell seeded, aligned fibre mats wrapped around them. The 3D constructs were cultured in a micro-bioreactor with perfusion the growth media transiently through the macro-porous tubing for 14 days. Experimental data confirm that 3D constructs from static and perfused cultures enhanced cell viability, uniform cell distribution and alignment due to nutrient provision from inside the 3D structure.

\section{ADVANTAGES AND LIMITATIONS FOR THE USE OF WJ-MSCS IN TISSUE ENGINEERING}

Results from pre-clinical and clinical trials with extraembryonic WJ-MSCs are still limited recent progress in basic and clinical research of these cells revealed multiple possibilities for their potential applications in regenerative medicine $[15,70]$. As mentioned earlier, use of the umbilical cord allows for a rapid initial isolation of large numbers of multipotent WJ-MSCs, with an average amount of 400,000 cells per umbilical cord, avoiding the need for extensive multiplication and potential epigenetic damage [42]. Such amounts of perinatal MSCs are much bigger than the average amounts of postnatal MSCs normally obtained from human bone marrow [71, 72]. WJ-MSCs can also be successfully expanded for long-term use and differentiated in vitro. Furthermore, due to their close ontogenic relationship to embryonic stem cells, WJ-MSCs possess unique immunomodullatory characteristics. All these advantageous characteristics make WJ-MSCs very interesting and promising candidates for different fields of regenerative medicine (Fig. 4).

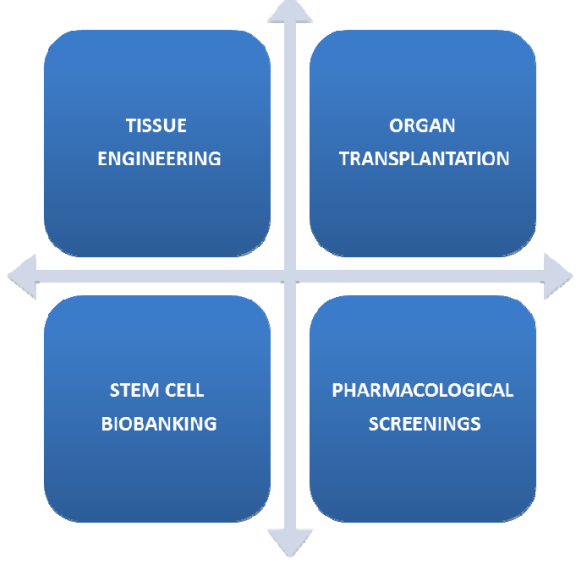

Fig. (4). Potential clinical directions for the use of WJ-MSCs.

Scaffold-based tissue engineering using WJ-MSCs could facilitate the construction of living autologous replacement structures such as vascular grafts and heart valves $[73,74]$.
In order to fabricate such cardio-vascular constructs, cells would be seeded onto biodegradable scaffolds that are implanted into a biomimetic system "bioreactor", where tissue formation and maturation would accelerate. After a defined time period, sufficient tissue formation have taken place and the constructs were ready for implantation [75]. Scaffold-free tissue engineering based on WJ-MSCs could facilitate stem cell based structures, such as stem cell sheets or micro-tissues, with great potential for the wound healing $[76,77]$. Due to WJ-MSCs' unique immuno-modulatory characteristics, such scaffold-free structures even could have potential for use in allogenic transplantations. Currently, the first attempts to use perinatal placenta-derived stem cells (PD-MSCs) for the manufacturing of live, differentiable cell sheets have been successfully demonstrated $[78,79]$.

The history of biobanking started from $1990^{\text {th }}$ when first banks for storage of UCB were established. Because of a strong biomedical need, stem cell biobanking has led to a growth in stem cell applications [80]. However, umbilical cord blood, containing both hematopoietic and mesenchymal stem cells, is still a predominant source of extra-embryonic MSCs useful for cell biobanking first attempts to store samples of umbilical cords itself have been currently performed. Comparison studies of different MSC sources have shown that WJ-MSCs have significant potential for cell biobanking [81]. In the concept of umbilical cord biobanking samples containing WJ-MSCs could be taken from the placenta post-delivery, frozen, and stored for the long term. On demand, such samples can be thawed, and isolated MSCs can be transplanted into the patient.

Though WJ-MSCs have potential in in vivo applications, they can be immediately utilised in pharmacological screening. Presently, many efforts will be performed for development of different cell-based systems as test-objects for determining various drug-related or physiologically mediated effects. Until recently, attempts to use human MSCs for cellbased assays have been hampered by the lack of appropriate transfection methods. Employing of new transfecting systems allow transfection of MSCs with high efficiency without affecting of their functionality. Systems based on single transfected WJ-MSCs, as well as its assemblies, could be utilised to determine different pharmacological, toxicological or pathological effects in perinatal medicine, or instance.

Additional specific field for applications of WJ-MSCs is transplantation of solid organs. It has been demonstrated, that upon systematic administration ex vivo expanded MSCs preferentially homed into damaged tissues and participate in regeneration processes through production of specific paracrine factors [12]. As was mentioned above MSCs are able to modulate / suppress immunologic response through interactions with different immune cells. Currently performed investigations indicate that MSCs contribute in inhabitation of rejection and prevention / controlling of GVHD after HSC transplantation. The first report about the potential of bone marrow MSCs for treatment GVHD in 9year old boy who received a matched unrelated donor HSC transplant has been published in 2004 [82]. As was mentioned above WJ-MSCs as well as other perinatal extraembryonic MSCs possess advantageous immuno-suppressive 
potential, and those can be seen as promising candidates for immunomodulation and treatment of GVHD.

Despite knowledge of its advanced characteristics and first reports of successful pre-clinical and clinical applications, WJ-MSCs require further study to determine its clinical limitations and establish realistic clinical protocols. Firstly, it is unknown if WJ-MSCs engraft in the long-term and display self-renewal and multipotency in vivo. Secondly, the amount of WJ-MSCs required for successful clinical applications is huge therefore the problem of scale-up is very important for their successful use in tissue regeneration. For this reason, establishing parameters for (I) GMP-related expansion of sufficient cell amounts and (II) routine clinical applications of GMP-expanded cells are two critical aspects to consider for the prospective transplantation of WJ-MSCs. First attempts to establish critical parameters for generation of clinically-sufficient amounts of WJ-MSCs have been performed only recently [83-85]. Parameters for applications of stem cells on human patients are summarized in the polices of the world marrow donor association (WMDA) as well as in the international standards for cellular therapy, product collection, processing and administration. Currently they are defined for hematopoietic progenitor cells (HPC), human cells, tissues or cellular or tissue-based products (HCT/Ps), and cord blood units (CBU). These parameters include infection disease markers (such as hepatitis B and $\mathrm{C}$, human HIV, HTLV, CMV, syphilis), number of pregnancies, and number of transfusions, for instance. Moreover, successful transplantation is strongly related to the histocompatibility of donor and recipient. Therefore, analysis of donor histocompatibility (specifically for HLA-A, B, C, DRB1, $\mathrm{DRB} 3$, and DQB1) is the most important criterion in bone marrow and PBSC transplantation. Unlike HSCs and HCT/ Ps, parameters for routine clinical applications of human adult and extra-embryonic MSCs are not yet established. Currently, only the minimal criteria for defining multipotent mesenchymal stromal cells, mentioned above, were summarized as a position statement from the International Society for Cellular Therapy (ISCT) [49]. Thirdly, replacements currently applicable in tissue scaffold-based tissue engineering are mostly based on foreign materials, such as natural, synthetic or hybrid polymers, which result in a lack of growth and remodelling and carry the risks for thrombo-embolic complications and infections. Possible problems concerning these systems are systemic toxicity, growth limitation, differentiation and function restraints, incorporation barriers and cell or tissue delivery difficulties. Thus, the development of compatible biomaterials that do not mitigate $\mathrm{WJ}$ MSC regenerative- and immuno-modulatory-potential is necessary.

\section{CONCLUSIONS}

Stem cell based regenerative medicine can be seen as a novel revolution approach for treatment of reconstruction of damaged human tissues and organs as well as broad range of diseases, such as congenital abnormalities and inherent pathologies. Therefore identification and characterization of novel stem cell sources is critical for clinical success of regenerative medicine. Emerging evidence suggests that different compartments of the human placenta are versatile sources of mesenchymal stem / stromal cells, called extra- embryonic MSCs. These extra-embryonic MSCs, derived from Wharton's jelly, are assigned an intermediate position between pluripotent embryonic stem cells (ESCs) and multipotent adult MSCs, and share specific properties both of these cell types. Due to their close ontogenetic relationship with ESCs, extra-embryonic WJ-MSCs possess extended plasticity, self-renewal capacity, and long-term expansion potential as well as immunoprivileged characteristics comparable with post-natal MSCs. The isolation of extraembryonic WJ-MSCs is non-invasive and poses no potential risk for the patient. In addition, extra-embryonic tissues represent so called "waste material", which is normally discarded after birth; their isolation is not associated with any current ethical concerns. Experimental results of last decade have shown that WJ-MSCs have great potential in tissue engineering, in which one of the most promising directions for their use is cardio-vascular tissue engineering. Nonetheless, long term survival of the stem cells, in the host tissue after transplantation and establishment of treatment regimen are critical issues which still hampering broad clinical applications of WJ-MSCs. For this reason the establishment of clinically relevant criteria for isolation, characterization, long-term cultivation, and maintenance of human MSCs is absolutely necessary for the successful use of WJ-MSCs in regenerative medicine applications.

\section{ACKNOWLEDGEMENT}

None declared.

\section{CONFLICT OF INTEREST}

None declared.

\section{REFERENCES}

[1] Da Silva Meirelles L, Chagastelles PC, Nardi NB. Mesenchymal stem cells reside in virtually all post-natal organs and tissues. J Cell Sci 2006; 119: 2204-13.

[2] Caplan AI. Mesenchymal stem cells. J Orthop Res 1991; 9: 641-50.

[3] Da Silva Meirelles L, Caplan AI, Nardi NB. In search of the in vivo identity of mesenchymal stem cells. Stem Cells 2008; 26: 2287-99.

[4] Rastegar F, Shenaq D, Huang J, et al. Mesenchymal stem cells: Molecular characteristics and clinical applications. World J Stem Cells 2010; 2: 67-80

[5] Friedenstein AJ, Deriglasova UF, Kulagina NN, et al. Precursors for fibroblasts in different populations of hematopoietic cells as detected by the in vitro colony assay method. Exp Hematol 1974; 2: 83-92.

[6] Ma T. Mesenchymal stem cells: From bench to bedside. World J Stem Cells. 2010; 2 : 13-17.

[7] Tuan RS, Boland G, Tuli R. Adult mesenchymal stem cells and cell-based tissue engineering. Arthritis Res Ther 2003; 5: 32-45.

[8] Stagg J, Galipeau J. Immune plasticity of bone marrow-derived mesenchymal stromal cells. Handb Exp Pharmacol 2007: 45-66.

[9] Noel D, Djouad F, Bouffi C, Mrugala D, Jorgensen C. Multipotent mesenchymal stromal cells and immune tolerance. Leuk Lymphoma 2007; 48: 1283-9.

[10] Ramasamy R, Fazekasova H, Lam EW, Soeiro I, Lombardi G, Dazzi F. Mesenchymal stem cells inhibit dendritic cell differentiation and function by preventing entry into the cell cycle. Transplantation 2007; 83: 71-6.

[11] Nauta AJ, Fibbe WE. Immunomodulatory properties of mesenchymal stromal cells. Blood 2007; 110: 3499-506.

[12] Hematti P. Role of mesenchymal stromal cells in solid organ transplantation. Transplant Rev 2008; 22: 262-73. 
[13] Zhang X, Jiao C, Zhao S. Role of mesenchymal stem cells in immunological rejection of organ transplantation. Stem Cell Rev 2009; 5: 402-9.

[14] Marcus AJ, Woodbury D. Fetal stem cells from extra-embryonic tissues: Do not discard. J Cell Mol Med 2008; 12: 730-42.

[15] Pappa KI, Anagnou NP. Novel sources of fetal stem cells: Where do they fit on the developmental continuum? Regen Med 2009; 4: 423-33.

[16] Semenov OV, Koestenbauer S, Riegel M, et al. Multipotent mesenchymal stem cells from human placenta: Critical parameters for isolation and maintenance of stemness after isolation. Am J Obstet Gynecol 2010; 202: 191-3.

[17] Ilancheran S, Moodley Y, Manuelpillai U. Human fetal membranes: A source of stem cells for tissue regeneration and repair? Placenta 2009; 30: 2-10.

[18] Strakova Z, Livak M, Krezalek M, Ihnatovych I. Multipotent properties of myofibroblast cells derived from human placenta. Cell Tissue Res 2008; 332: 479-88.

[19] Markov V, Kusumi K, Tadesse MG, et al. Identification of cord blood-derived mesenchymal stem/stromal cell populations with distinct growth kinetics, differentiation potentials, and gene expression profiles. Stem Cells Dev 2007; 16: 53-73.

[20] De Coppi P, Bartsch G, Jr., Siddiqui MM, et al. Isolation of amniotic stem cell lines with potential for therapy. Nat Biotechnol 2007; 25: 100-6.

[21] Tsai MS, Lee JL, Chang YJ, Hwang SM. Isolation of human multipotent mesenchymal stem cells from second-trimester amniotic fluid using a novel two-stage culture protocol. Hum Reprod 2004; 19: 1450-6.

[22] Gluckman E, Rocha V. History of the clinical use of umbilical cord blood hematopoietic cells. Cytotherapy 2005; 7: 219-27.

[23] Rocha V, Locatelli F. Searching for alternative hematopoietic stem cell donors for pediatric patients. Bone Marrow Transplant 2008; 41: 207-14.

[24] Langer R, Vacanti JP. Tissue engineering. Science 1993; 260: 9206.

[25] Langer R, Vacanti JP, Vacanti CA, Atala A, Freed LE, VunjakNovakovic G. Tissue engineering: Biomedical applications. Tissue Eng 1995; 1: 151-61.

[26] Gude NM, Roberts CT, Kalionis B, King RG. Growth and function of the normal human placenta. Thromb Res 2004; 114: 397-407.

[27] Larsen WJ. Human embryology. Chirchill Livingstone, Philadelphia 2001

[28] Benirschke K, Kaufmann, P., Baergen, RN. Pathology of the human placenta. Springer Berlin/Heidelberg, $5^{\text {th }}$ ed. 2006.

[29] Huppertz B. The anatomy of the normal placenta. J Clin Pathol 2008; 61: 1296-302.

[30] Kaufmann P. Basic morphology of the fetal and maternal circuits in the human placenta. Contrib Gynecol Obstet 1985; 13: 5-17.

[31] Raio L, Ghezzi F, Di Naro E, et al. Sonographic measurement of the umbilical cord and fetal anthropometric parameters. Eur J Obstet Gynecol Reprod Biol 1999; 83: 131-5.

[32] Di Naro E, Ghezzi F, Raio L, Franchi M, D'Addario V. Umbilical cord morphology and pregnancy outcome. Eur J Obstet Gynecol Reprod Biol 2001; 96: 150-7.

[33] Sobolewski K, Bankowski E, Chyczewski L, Jaworski S. Collagen and glycosaminoglycans of wharton's jelly. Biol Neonate 1997; 71: $11-21$.

[34] Vizza E, Correr S, Goranova V, et al. The collagen skeleton of the human umbilical cord at term. A scanning electron microscopy study after 2n-naoh maceration. Reprod Fertil Dev 1996; 8: 885-94.

[35] Kobayashi K, Kubota T, Aso T. Study on myofibroblast differentiation in the stromal cells of wharton's jelly: Expression and localization of alpha-smooth muscle actin. Early Hum Dev 1998; 51: 223-33.

[36] Baudin B, Bruneel A, Bosselut N, Vaubourdolle M. A protocol for isolation and culture of human umbilical vein endothelial cells. Nat Protoc 2007; 2: 481-5.

[37] Murohara T. Cord blood-derived early outgrowth endothelial progenitor cells. Microvasc Res 2010; 79: 174-7.

[38] Schiebler T, Kaufmann P. Reife plazenta. In: V B, Th S, F K, eds. Die plazenta des menschen. Stuttgart: Georg Thieme Verlag 1981: 51-94.

[39] Thomas J. Introduction to human embryology. London 1968.

[40] Wynn R. Principles of placentation and early human development. In: Gruenwald P, Ed. The placenta. London: MTP; 1975: 18-35.
[41] Troyer DL, Weiss ML. Wharton's jelly-derived cells are a primitive stromal cell population. Stem Cells 2008; 26: 591-9.

[42] Karahuseyinoglu S, Cinar O, Kilic E, et al. Biology of stem cells in human umbilical cord stroma: In situ and in vitro surveys. Stem Cells 2007; 25: 319-31.

[43] Nanaev AK, Kohnen G, Milovanov AP, Domogatsky SP, Kaufmann P. Stromal differentiation and architecture of the human umbilical cord. Placenta 1997; 18: 53-64.

[44] Can A, Karahuseyinoglu S. Concise review: Human umbilical cord stroma with regard to the source of fetus-derived stem cells. Stem Cells 2007; 25: 2886-95.

[45] La Rocca G, Anzalone R, Corrao S, et al. Isolation and characterization of oct-4+/hla-g + mesenchymal stem cells from human umbilical cord matrix: Differentiation potential and detection of new markers. Histochem Cell Biol 2009; 131: 267-82.

[46] Asakawa N, Shimizu T, Tsuda Y, et al. Pre-vascularization of in vitro three-dimensional tissues created by cell sheet engineering. Biomaterials 2010; 31: 3903-9.

[47] Seshareddy K, Troyer D, Weiss ML. Method to isolate mesenchymal-like cells from wharton's jelly of umbilical cord Methods Cell Biol 2008; 86: 101-19.

[48] Solanki A, Kim JD, Lee KB. Nanotechnology for regenerative medicine: Nanomaterials for stem cell imaging. Nanomedicine (Lond) 2008; 3: 567-78.

[49] Dominici M, Le Blanc K, Mueller I, et al. Minimal criteria for defining multipotent mesenchymal stromal cells. The international society for cellular therapy position statement. Cytotherapy 2006; 8: $315-7$.

[50] Rojewski MT, Weber BM, Schrezenmeier H. Phenotypic characterization of mesenchymal stem cells from various tissues. Transfus Med Hemother 2008; 35: 168-84.

[51] Anzalone R, Lo Iacono M, Loria T, et al. Wharton's jelly mesenchymal stem cells as candidates for beta cells regeneration: Extending the differentiative and immunomodulatory benefits of adult mesenchymal stem cells for the treatment of type 1 diabetes. Stem Cell Rev 2011; 7: 342-63.

[52] Anzalone R, Lo Iacono M, Corrao S, et al. New emerging potentials for human wharton's jelly mesenchymal stem cells Immunological features and hepatocyte-like differentiative capacity. Stem Cells Dev 2010; 19: 423-38.

[53] Mayer J. Uses of homograft conduits for right ventricle to pulmonary artery connections in the neonatal period. Semin Thorac Cardiovasc Surg 1995; 7.

[54] Schoen FJ, Levy RJ. Tissue heart valves: Current challanges and future research perspectives. J Biomed Mater Res 1999; 47: 43965.

[55] Turner CG, Fauza DO. Fetal tissue engineering. Clin Perinatol 2009; 36: 473-88.

[56] Zund G, Hoerstrup SP, Schoeberlein A, et al. Tissue engineering: A new approach in cardiovascular surgery; seeding of human fibroblasts followed by human endothelial cells on resorbable mesh. Eur J Cardiothorac Surg 1998; 13: 160-4.

[57] Lehle K, Hoenicka M, Jacobs VR, Schmid FX, Birnbaum DE. Cryopreservation of human endothelial cells for vascular tissue engineering. Cryobiology 2005; 50: 154-61.

[58] Schmidt D, Breymann C, Weber A, et al. Umbilical cord blood derived endothelial progenitor cells for tissue engineering of vascular grafts. Ann Thorac Surg 2004; 78: 2094-8.

[59] Horstrup S, Kadner A, Breymann C. Living, autologous pulmonary artery conduits tissue engineered from human umbilical cord cells. Ann Thor Surg 2002; 74: 46-52.

[60] Kadner A, Hoerstrup S, Tracy J, et al. Human umbilical cord cells: A new cell source for cardiovascular tissue engineering. Ann Thorac Surg 2002; 74: 1422-8.

[61] Breymann C, Schmidt D, Hoerstrup SP. Umbilical cord cells as a source of cardiovascular tissue engineering. Stem Cell Rev 2006; 2: 87-92.

[62] Kadner A, Zund G, Maurus C, et al. Human umbilical cord cells for cardiovascular tissue engineering: A comparative study. Eur J Cardiothorac Surg 2004; 25: 635-41.

[63] Schmidt D, Mol A, Neuenschwander S, et al. Living patches engineered from human umbilical cord derived fibroblasts and endothelial progenitor cells. Eur J Cardiothorac Surg 2005; 27: 795-800. 
[64] Schmidt D, Mol A, Breymann C, et al. Living autologous heart valves engineered from human prenatally harvested progenitors. Circulation 2006; 114: 125-31

[65] Schmidt D, Mol A, Odermatt B, et al. Engineering of biologically active living heart valve leaflets using human umbilical cordderived progenitor cells. Tissue Eng 2006; 12: 3223-32.

[66] Shinoka T, Ma PX, Shum-Tim D, et al. Tissue engineered heart valves. Autologous valve leaflet replacement study in a lamb model. Circulation 1996; 94 (9 Suppl): 164-8.

[67] Penolazzi L, Tavanti E, Vecchiatini R, et al. Encapsulation of mesenchymal stem cells from wharton's jelly in alginate microbeads. Tissue Eng Part C Methods 2010; 16: 141-55.

[68] Zimmermann U, Cramer, H, Jork, A, et al. Microencapsulationbased cell therapy, in biotechnology: Special processes. WileyVCH Verlag GmbH, Weinheim, Germany 2008; Volume 10.

[69] Kenar H, Kose GT, Toner M, Kaplan DL, Hasirci V. A 3d aligned microfibrous myocardial tissue construct cultured under transient perfusion. Biomaterials 2011.

[70] Parolini O, Alviano F, Bagnara GP, et al. Concise review: Isolation and characterization of cells from human term placenta: Outcome of the first international workshop on placenta derived stem cells. Stem Cells 2008; 26: 300-11.

[71] Castro-Malaspina H, Gay RE, Resnick G, et al. Characterization of human bone marrow fibroblast colony-forming cells (cfu-f) and their progeny. Blood 1980; 56: 289-301.

[72] Lazarus HM, Haynesworth SE, Gerson SL, Rosenthal NS, Caplan AI. Ex vivo expansion and subsequent infusion of human bone marrow-derived stromal progenitor cells (mesenchymal progenitor cells): Implications for therapeutic use. Bone Marrow Transplant 1995; 16: 557-64.

[73] Schmidt D, Hoerstrup SP. Tissue engineered heart valves based on human cells. Swiss Med Wkly 2007; 137 (Suppl 155): 80S-5S.

[74] Schmidt D, Mol A, Kelm JM, Hoerstrup SP. In vitro heart valve tissue engineering. Methods Mol Med 2007; 140: 319-30.

[75] Hoerstrup SP, Sodian R, Sperling JS, Vacanti JP, Mayer JE, Jr. New pulsatile bioreactor for in vitro formation of tissue engineered heart valves. Tissue Eng 2000; 6: 75-9.
[76] Kelm JM, Lorber V, Snedeker JG, et al. A novel concept for scaffold-free vessel tissue engineering: Self-assembly of microtissue building blocks. J Biotechnol 2010; 148: 46-55

[77] Yang J, Yamato M, Shimizu T, et al. Reconstruction of functional tissues with cell sheet engineering. Biomaterials 2007; 28: 5033-43.

[78] Guillaume-Gentil O, Semenov OV, Zisch AH, Zimmermann R, Voros J, Ehrbar M. Ph-controlled recovery of placenta-derived mesenchymal stem cell sheets. Biomaterials 2011; 32: 4376-84.

[79] Semenov OV, Malek A, Bittermann AG, Voros J, Zisch AH. Engineered polyelectrolyte multilayer substrates for adhesion, proliferation, and differentiation of human mesenchymal stem cells. Tissue Eng Part A 2009; 15: 2977-90.

[80] Engel E, Michiardi A, Navarro M, Lacroix D, Planell JA. Nanotechnology in regenerative medicine: The materials side. Trends Biotechnol 2008; 26: 39-47.

[81] Cavallo C, Cuomo C, Fantini S, et al. Comparison of alternative mesenchymal stem cell sources for cell banking and musculoskeletal advanced therapies. J Cell Biochem 2011; 112: 1418-30.

[82] Le Blanc K, Rasmusson I, Sundberg B, et al. Treatment of severe acute graft-versus-host disease with third party haploidentical mesenchymal stem cells. Lancet 2004; 363: 1439-41.

[83] Nekanti U, Mohanty L, Venugopal P, Balasubramanian S, Totey S, $\mathrm{Ta}$ M. Optimization and scale-up of wharton's jelly-derived mesenchymal stem cells for clinical applications. Stem Cell Res 2010; $5: 244-54$

[84] Nekanti U, Dastidar S, Venugopal P, Totey S, Ta M. Increased proliferation and analysis of differential gene expression in human wharton's jelly-derived mesenchymal stromal cells under hypoxia. Int J Biol Sci 2010; 6: 499-512.

[85] Nekanti U, Rao VB, Bahirvani AG, Jan M, Totey S, Ta M. Longterm expansion and pluripotent marker array analysis of wharton's jelly-derived mesenchymal stem cells. Stem Cells Dev 2010; 19: 117-30.

(C) Semenov and Breymann; Licensee Bentham Open.

This is an open access article licensed under the terms of the Creative Commons Attribution Non-Commercial License (http: //creativecommons.org/licenses/by$\mathrm{nc} / 3.0 /$ ), which permits unrestricted, non-commercial use, distribution and reproduction in any medium, provided the work is properly cited. 